\title{
Introduction of a redundant actuator using planetary gear trains for human centred robotics
}

\author{
Stein Crispel*, Pablo López-García, Tom Verstraten, Elias Saerens and Dirk Lefeber
}

\begin{abstract}
All authors are with the Robotics and Multibody Mechanics Research Group (R\&MM), Faculty of Mechanical Engineering, Vrije Universiteit Brussel (VUB) and Flanders Make, Pleinlaan 2, 1050 Elsene, Belgium.
\end{abstract}

\begin{abstract}
Matching motor efficiency and performance with the load demands can significantly improve the overall efficiency of a driveline. Inspired by the automotive sector -with the high interest of hybrid and electric cars currently-, the authors have studied how state of the art technologies can be used in the relatively new field of collaborative and Human centred robotics. Multiple transmission systems have been considered, among others redundant actuators (both static and kinematic) and continuously variable transmissions. Based on these findings and the experience of the research group on customised planetary gear trains for Human Limb Assistance and Replication, an extensive review of existing redundant actuators is presented in combination with an alternative transmission system which does not need any auxiliary gear transmissions and hence can be lighter and more compact than state of the art drivelines for Human centred robotics. A calculation was performed -including the efficiency model presented by Müller- which shows the high potential of this type of dual-motor actuator.
\end{abstract}

\section{Introduction}

At nominal conditions, electric motors can achieve very high efficiencies. This partially explains why they are used in many machines and industries. In several applications, these motors operate at a constant speed and load, allowing the engineer to optimise the entire driveline for this specific operation. As a result, the efficiency of brushless DC motors can reach up to 85$90 \%$ [1]. However, when the motor's speed and torque change, the efficiency can drop drastically [2]. This is a typical problem in robotics, where actuators are used in highly fluctuating operating conditions (Figure 1). As a result, some working points will be situated in poor efficiency regions, leading to a high energy consumption. A good example of this issue can be found in the MIT Cheetah robot, where up to $68 \%$ of the total energy consumption is consumed by heating of the motor windings (i.e. Joule losses) [3].

Matching motor efficiency and performance with the load demands can significantly improve the overall efficiency of a driveline. This can be achieved by means of a well-chosen transmission. By trading speed for torque and vice versa, i.e. changing the gear ratio, the working point of the motor can be placed in a more energy-efficient region (Figure 1). However, transmissions with one fixed reduction can only be optimised for a specific task and their efficiency drops when the working points are widely spread. If this is the case, variable transmissions can be a solution. The most

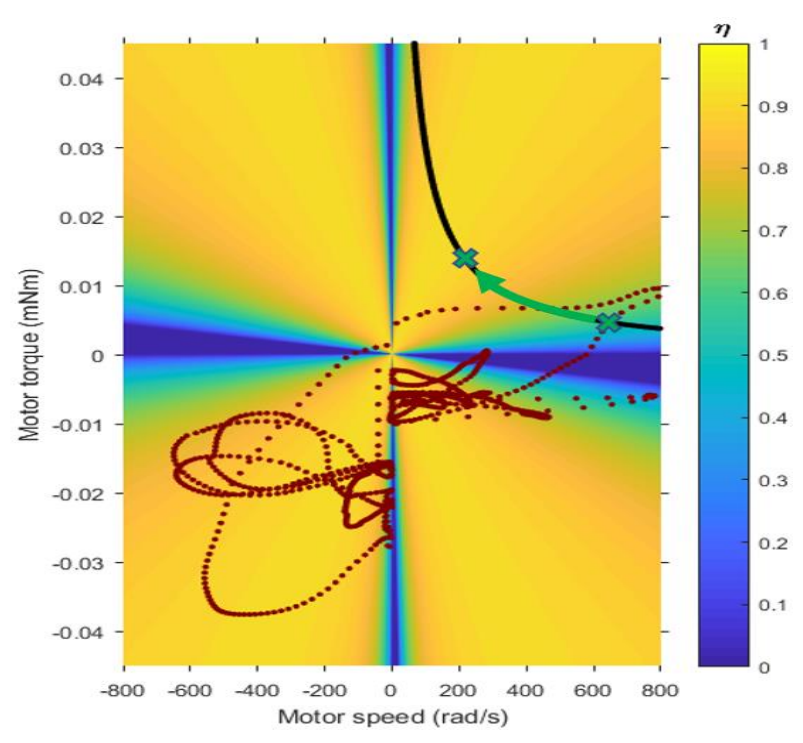

Fig. 1. Motor working points (red) on the theoretical efficiency map for a stiff actuator placed in the elbow joint of a warehouse robot [32]. By changing the gear ratio, speed can be traded for torque. This is depicted by the green working point, which is shifted to a higher-efficiency region on the black constant-power line.

well know variable transmissions are the Continuously Variable Transmissions (CVTs) [4]-[12]. However, since they often use two motors -one to drive the load and the other to change the gear ratio- they are actually a subcategory of Dual Motor Actuators (DMAs) [13][15]. Both concepts are discussed in more detail in section 2 .

* Corresponding author: Stein.Crispel@vub.be 
As will be discussed further current DMAs -which are often designed for the automotive sector- are often too large and heavy or have a too low operation range or output torque to use in robotic applications. Therefore, this paper will discuss a dual motor actuator which is based on the Wolfrom Planetary Gear Train (PGT). One advantage of this type of PGT is the very high gear ratio it can generate which can even go above 1:1000 for only a two-stage assembly [16]-[18], and hence can remain very compact.

The paper is organized as follows: First, in section 2 an extensive overview of DMAs and CVTs is discussed with the focus on the field of robotics. Secondly, in section 3 a theoretical analysis is provided for a Wolfrom-based DMA, this analysis comprises a kinematic and load analysis of the entire gearbox, together with the derivation of an analytical efficiency model. To end this paper a conclusion and future work is given in section 4 .

\section{Overview of DMAs and CVTs}

\subsection{Continuously variable transmissions}

Although there are several types of CVTs, the most common are all based on the same fundamental principle: two shafts with a different diameter are connected by a frictional element. By varying their diameter ratio, a different gear ratio is obtained between the input motor and output (Figure 2). In order to change the diameter ratio an auxiliary actuator is often required.

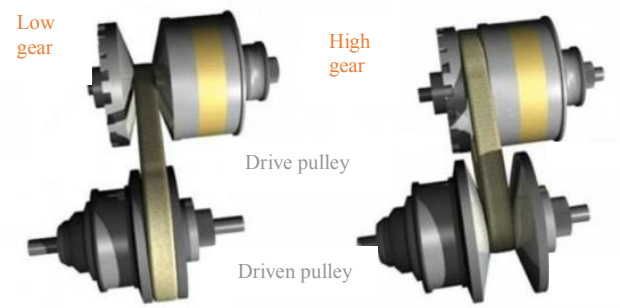

Fig. 2. Concept of the pulley based CVT in low(left) and high(right) gear ratio [33].

\subsubsection{CVTs in the automotive industry}

Most CVTs can be found in the automotive industry, where over the past 50 years different concepts have been investigated. These have shown major benefits in terms of energy efficiency and increased performance [4]. The most common CVTs use friction to transmit the torques from one shaft to another. Representative examples are the belt/chain CVT [11], Toroidal CVT [5], [6], Spherical CVT [9], Ball-type CVT [10], etc. In applications where very high powers need to be converted, hydrostatic CVTs are also used [19]. Within friction based CVTs, the ones using a belt or chain are the most commonly used due to their simple construction, easy maintenance [20] and high efficiency in comparison with the other systems. Examples of car manufacturers who have developed systems based on the latter concept are Nissan with the XTRONIC CVT [21] and Toyota with the $\mathrm{K}$ series.

\subsubsection{CVTs in robotics}

Although these concepts are suitable for the automotive sector, they are not adopted in robotics due to the difference in requirements, which cannot be met by current CVTs. The three main problems can be summarised as follows. First, CVTs for the automotive industry are based on friction or hydrostatics, hence they cannot be downscaled in order to fit the demanding mass and size requirements of robotic applications while still providing the required torque. Secondly, the classical CVTs have issues with rapid changes in gear ratio [11]. However, the dynamic character of robotic actuation is very high, i.e. speed is changing very fast and continuously [22]. Thirdly, the typical gear ratio of CVTs in the automotive industry is in the order of 0.5 to 2 [4], while Robotic applications require up to 200 times this value. Hence, additional systems, such as lever arms and gearboxes, are required. This leads to more complex and heavier actuators. More compact CVTs have been developed [6]-[8], [12], but at the cost of a decreased operation range, lower output torque or efficiency. Consequently, all the limitations on current CVTs, most importantly the gear ratio range, make state-of-the-art systems unsuitable for robotic actuators.

\subsection{Dual motor actuators (DMAs)}

Another way to obtain a Variable Transmission is by combining two motors. By using a second motor an extra degree of freedom is created, which can be used to meet a secondary objective. This redundancy can be used to maximise e.g. energy efficiency [13], reduced motor torques, increasing acceleration capability (even at high torques) and minimizing impedance [23].

\subsubsection{Kinematically redundant DMAs}

A tool to couple two motors is by using the two Degrees Of Freedom (DOFs) of a Planetary Gear Transmission (PGT. In classical PGTs, the ring gear is fixed to the housing. By doing so, one kinematic degree of freedom is used, resulting in a Single Input-Single Output (SISO) system. Therefore, an extra kinematic DOF can be obtained when a second motor is used to actuate this ring (Figure 3). This layout is often referred to as differential or summing device since the output speed is a linear combination of the input speeds [24]. However, this comes at the cost that the torques of the two motors are coupled, i.e. at steady state, the forces in the planet-ring interface and planet-sun interface should be equal. The differential concept has been studied in [13]-[15], [25] and it is suggested that it has a great potential in reducing the energy for highly dynamical applications [23]. For example, the preliminary study in [26] has shown that the energy consumption of an active ankle 
could be reduced by $16 \%$ when a DMA is used. Moreover, this system allows high torques to be transmitted without the risk of slipping since the forces are transferred through rigid teeth.

A downside is that only low gear ratio changes can be obtained by this layout, typically 3 to 8 . Hence,

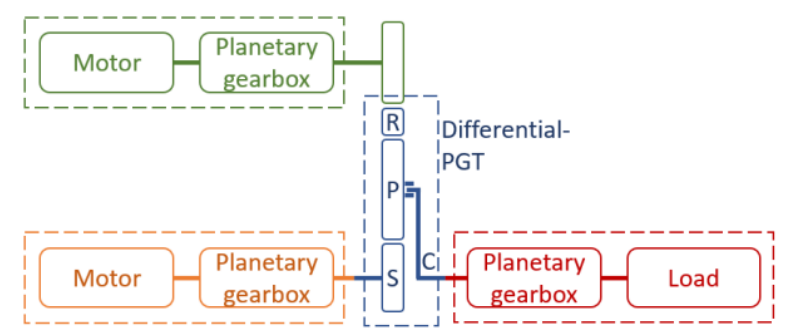

Fig. 3. Planetary gear transmission used as differential device.The output speed (C) is a linear combination of the speeds of ring gear $(\mathrm{R})$ and sun gear $(\mathrm{S})$.

several additional auxiliary gearboxes (up to three, Figure 3.) are required to reach the required output torques and operation range. This makes that the overall system becomes bulky and heavy, while the mechanical efficiency is reduced.

\subsubsection{Statically redundant DMAs}

A different manner of obtaining an extra DOF is by adding an extra motor in parallel. A simplified model of this concept, which can be called a statically redundant DMA, is given in Figure 4. Here, the speed ratio of the shafts of the two motors is constant. On the other hand, an infinite amount of input torques combinations can be found to match the output torque. Studies have shown that this configuration has a good control bandwidth and excellent torque reliability [27]. Moreover, torque distortion, peak impact torque and energy efficiency are improved compared to a single motor configuration [27], [28]. However, these studies conclude that more compact concepts should be designed to remove the extra gearboxes which are still required in this system, resulting in the same problems as in 2.2.1.

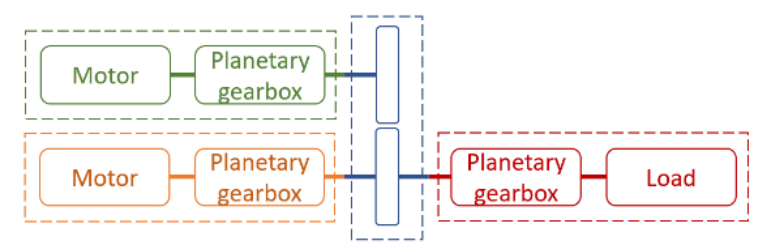

Fig. 4. Possible configurations where the input torques of the motor add up. Hence, adding a static DOF.

\subsubsection{Combination of statically and kinematically redundant actuators}

To freely distribute both the torques and speeds among the actuators, kinematically and statically redundant configurations can be combined. This has been done in the Toyota Prius, where the traditional combustion motor is combined with two electric motors/generators. According to [29] this system is more efficient, smoother and can produce higher accelerations than mechanical CVTs. The static-kinematic actuation can also be used to mimic the human muscle. An artificial equivalent of such a muscle, the "discrete muscle-like actuator", has been studied in [30] where it is concluded that this principle can be expected to work well in tasks typically done by humans.

\section{Introduction of Wolfrom DMA}

The architecture of a Wolfrom PGT is shown in Figure 5 , where generally the sun gear acts as the input and the ring $b$ as the output of the PGT. In this layout, the ring a is grounded and hence one kinematic degree of freedom is used. Indeed, when this gear is not allowed to rotate, the gear ratio between the output on ring $-\mathrm{Rb}$ - and the input on the sun -S- is uniquely defined. However, it is possible to attach a secondary motor to the first ring -Ra-

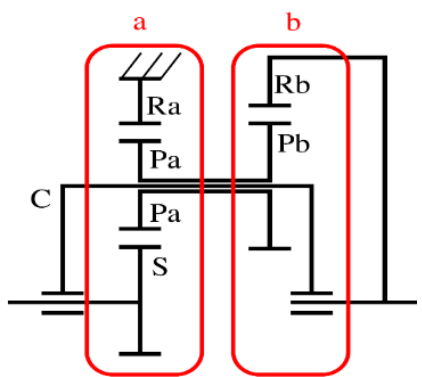

Fig. 5. Layout of a Wolfrom PGT. Stage a consists of a sun $\mathrm{S}-$, planetary -Pa- and ring -Ra- gear, of which the latter is grounded. Stage $\mathrm{b}$ is made of a second planetary $-\mathrm{Pb}$ - and ring $-\mathrm{Rb}$ - gear. Both stages are connected through the compound , resulting in a kinematic redundant actuator.

\subsection{Theoretical model}

\subsubsection{Speed relations of the Wolfrom}

Since the considered layout now has two kinematic degrees of freedom (K-DOFs), the output speed , $\omega_{R b}$, can be written as a linear combination of the sun, $\omega_{S}$, and ring a, $\omega_{R a}$, speeds:

$$
\left\{\begin{array}{l}
\omega_{R b}=\left(1-\frac{1}{i}\right) \omega_{R a}+\frac{1}{i} \omega_{S} \\
\omega_{c}=\left(1-\frac{1}{i_{0}}\right) \omega_{R a}+\frac{1}{i_{0}} \omega_{S}
\end{array}\right.
$$

Where $i$, the total gear ratio, is defined as:

$$
\left\{\begin{array}{l}
i_{0}=1+\frac{Z_{R a}}{Z_{S}} \\
i_{w}=\left(1-\frac{Z_{R a}}{Z_{P a}} \frac{Z_{P b}}{Z_{R b}}\right)^{-1} \\
i=i_{w} i_{0}
\end{array}\right.
$$


with $Z_{x}$ is the number of teeth of gear $x$.

Due to the two K-DOFs, two speeds need to be known to uniquely defne all other speeds of the system. However, to simplify the equations later on the redundancy factor, $\Omega \triangleq \frac{\omega_{S}-\omega_{R a}}{\omega_{R b}}$, will be used. As a consequence, the speeds can as a function of $\omega_{R b}$ and $\Omega$ :

$$
\left\{\begin{array}{l}
\omega_{S}=\left(1+\Omega-\frac{\Omega}{i}\right) \omega_{R b} \\
\omega_{R a}=\left(1-\frac{\Omega}{i}\right) \omega_{R b} \\
\omega_{C}=\left(1+\frac{\Omega}{i_{0}}-\frac{\Omega}{i}\right) \omega_{R b}
\end{array}\right.
$$

\subsubsection{Torque relation of the Wolfrom DMA}

In this configuration the meshing efficiency of the individual gear pairs have a strong effect on the total efficiency of the system. To integrate this important effect, the rolling power framework described by Müller [31] will be used. The main idea of this approach is that the total power of a gear in a PGT can be expressed as the sum of two separate powers. One is called the coupled power and the other is called rolling power. For gear $x$ they are defined respectively as:

$$
\left\{\begin{array}{l}
P_{x}^{\prime \prime}=T_{x} \omega_{c} \\
P_{x}^{\prime}=T_{x}\left(\omega_{x}-\omega_{c}\right) \triangleq T_{x} \omega_{x}^{\prime}
\end{array}\right.
$$

With $\omega_{c}$ the absolute rotational speed of the carrier and $T_{x}$ the torque on gear $x$. Hence, the rolling power can be seen as if the observer is fixed to the carrier.

Since there are no relative speeds in the coupled power between two gears -they all rotate at the same carrier speed-, the latter will not generate any meshing losses. Consequently, these losses can be determined by looking at only the rolling power.

The individual meshing efficiencies of the three contacts are given by following expression:

$$
\eta_{x}=\left\{\begin{array}{l}
-\frac{T_{p}^{(x)} \omega_{p}^{\prime}}{P_{x}^{\prime}} \text { when } P_{x}^{\prime}>0 \\
-\frac{P_{x}^{\prime}}{T_{p}^{(x)} \omega_{p}^{\prime}} \text { when } P_{x}^{\prime}<0
\end{array}\right.
$$

depending on the direction of the power going through the meshing. Where $\eta_{x}$ is the ordinary gear meshing efficiency and $T_{p}^{(x)}$ is the reaction torque on the planet gear $-\mathrm{Pa}$ or $\mathrm{Pb}$ - due to the torque of gear $x$. For example, $T_{p}^{(S)}$ represents the torque on planet $\mathrm{Pa}$, generated by the sun gear S. To simplify (5), the generalised efficiency function , $C_{x}$, is introduced [13]:

$$
C_{x}=-\frac{T_{p}^{(x)} \omega_{p}^{\prime}}{P_{x}^{\prime}}=\eta_{x}^{\operatorname{sign}\left(P_{x}^{\prime}\right)}
$$

In steady state -i.e. in the absence of acceleration-, the torque balance of the DMA is given by:

$$
T_{S}+T_{R a}+T_{R b}=0
$$

Similarly, the torque balance for the compound planetary gear can be expressed:

$$
T_{p}^{(S)}+T_{p}^{(R a)}+T_{p}^{(R b)}=0
$$

Which after some manipulation and the use of (4) and (6) can be written as:

$$
C_{S} \omega_{S}^{\prime} T_{S}+C_{R a} \omega_{R a}^{\prime} T_{R a}+C_{R b} \omega_{R b}^{\prime} T_{R b}=0
$$

Combining the two equations (7) and (9) and some manipulations of (1) result that the torques on the sunand ring gears are respectively:

$$
\left\{\begin{array}{l}
T_{S}=\frac{-i C_{R a}+\left(i-i_{0}\right) C_{R b}}{i\left[\left(i_{0}-1\right) C_{S}+C_{R a}\right]} T_{R b} \\
T_{R a}=\frac{i\left(1-i_{0}\right) C_{S}-\left(i-i_{0}\right) C_{R b}}{i\left[\left(i_{0}-1\right) C_{S}+C_{R a}\right]} T_{R b}
\end{array}\right.
$$

\subsubsection{Losses of the Wolfrom DMA}

Finally, the total losses, $P_{L}$, can be determined by expressing the power balance of the system:

$$
\begin{aligned}
P_{L} & =-\left(P_{S}+P_{R a}+P_{R b}\right) \\
& =-\frac{C_{S}\left(i_{0}-1\right)+C_{R a}(1-i)+C_{R b}\left(i-i_{0}\right)}{i\left[\left(i_{0}-1\right) C_{S}+C_{R a}\right]} \Omega P_{R b}
\end{aligned}
$$

Since this system has one static- and two kinematic degrees of freedom the power can be computed uniquely by the parameters $T_{R b}, \Omega$ and $\omega_{R b}$. Therefore, for each output, determined by a specific $T_{R b}$ and $\omega_{R b}$, an optimal $\Omega$ can be calculated to minimise the losses. However, this will be an iterative process since in order to calculate the torques using (10), the sign of the rolling powers should be known to determine $C_{x}$ which on its term needs the torques to be known.

\subsection{Case study}

To show the influence of redundancy factor $\Omega$, an example will be discussed in the following section. It is assumed that the ordinary meshing efficiencies of the two internal gear contacts are identical and equal to $99 \%$, while the efficiency of the external contact is equal to $98.5 \%$. The total gear ratio is equal to $70, i_{0}=8$ and the output torque is $100 \mathrm{Nm}$. To better show the influence of $\Omega$, the output efficiency $\eta_{\text {out }}$ is defined as:

$$
\eta_{\text {out }}=-\left(\frac{P_{S}+P_{R a}}{P_{R b}}\right)^{\operatorname{sign}\left(P_{R b}\right)}
$$


Note that this is not the conventual definition for the efficiency but that $P_{S}$ and $P_{R a}$ are always taken together. This definition is chosen to reflect better on how efficient the sun/ring a combination can produce/recuperate a certain power $P_{R b}$. For the considered example, the output efficiency is shown in

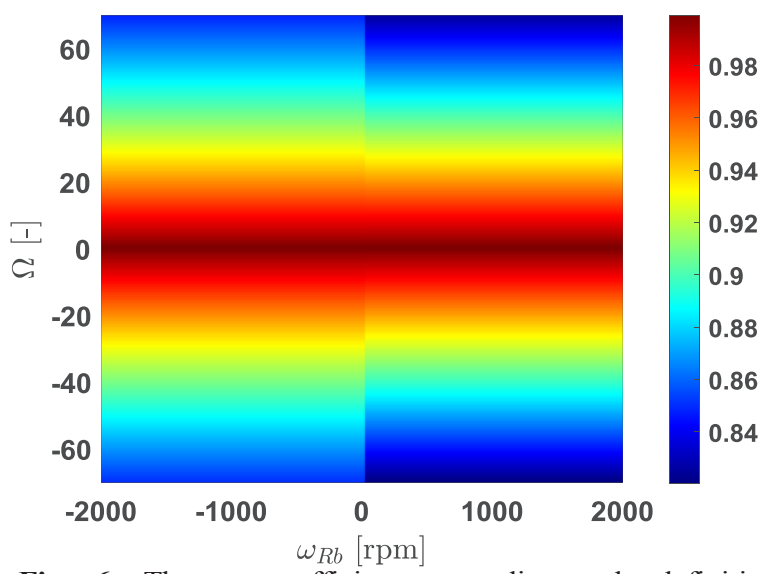

Fig. 6. The output efficiency according to the definition of (12) as a function of the output speed, $\omega_{\mathrm{Rb}}$, and the redundancy factor, $\Omega$.

Figure 6.

As can be seen the efficiency is only dependent on the sign of the speed of ring $\mathrm{b}, \omega_{R b}$, and linearly proportional with the redundancy factor, $\Omega$. In the same way it can be showed that this efficiency is also slightly dependent of the sign of the torque on ring $\mathrm{Rb}$.

It is also interesting to investigate the power distribution between the two inputs -the gears $\mathrm{S}$ and $\mathrm{Ra}-$. For the same example, the ratio $\frac{P_{R a}}{P_{S}}$ is depicted in Figure 7. As can be noticed, the sign of this ratio can be influenced by $\Omega$. I.e., it can be chosen that both motors combine their effort to drive the output or that one motor is in reverse drive while the other is in forward drive. This can be interesting for hybrid drivelines, where for example the combustion motor and an electric motor are combined to deliver peak power, but when less power is needed, the combustion engine can be used to charge the batteries through the electric motor.

\section{Conclusion and future work}

\subsection{Conclusion}

In this paper it has been shown that a high gear ratio Wolfrom PGT can be used as a component of a kinematically redundant DMA. As such it can lead to a more compact system than the current state-of-the-art solutions where extra gear boxes, etc. are required to produce the required torques. The output speed is then a linear combination of the two input speeds while their torques are linked. The efficiency of this DMA has been calculated using the efficiency model proposed by

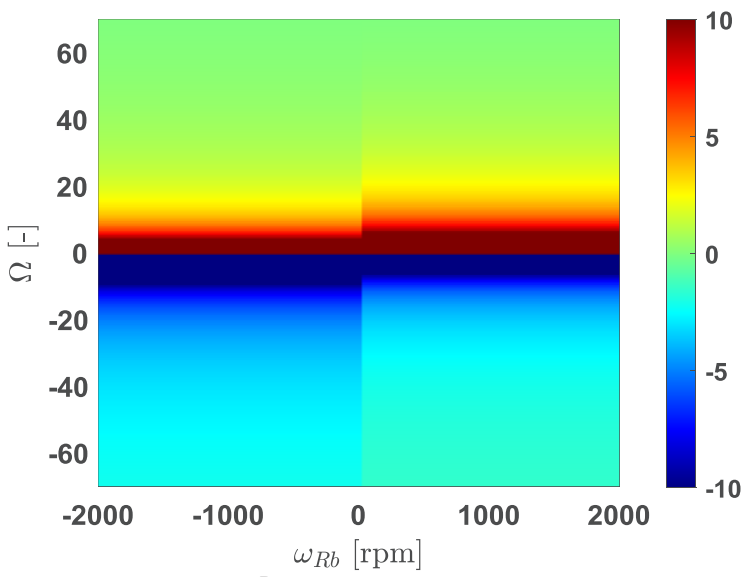

Fig. 7. The ratio $\frac{P_{R a}}{P_{s}}$ as a function of the output speed, $\omega_{R b}$, and the redundancy factor, $\Omega$.

Müller and a case study was presented. This showed that the efficiency is linear proportional with the redundancy factor.

\subsection{Future work}

Continuing on these results, further work will consist of including an efficiency model for the (electric-)motors and investigate how the redundancy can be exploited maximally in order to minimise the overall losses.

Stein Crispel, Elias Saerens and Tom Verstraten are all funded by the Research Foundation Flanders. Stein Crispel and Elias Saerens as SB PhD Fellows and Tom Verstraten as a Postdoctoral Fellow.

\section{References}

[1] A. Hughes, ELECTRIC MOTORS AND DRIVES. 1990.

[2] T. Verstraten, G. Mathijssen, R. Furnémont, B. Vanderborght, and D. Lefeber, "Modeling and design of geared DC motors for energy efficiency: Comparison between theory and experiments," Mechatronics, vol. 30, pp. 198-213, 2015, doi: 10.1016/j.mechatronics.2015.07.004.

[3] S. Seok, A. Wang, M. Y. Chuah, D. Otten, J. Lang, and S. Kim, "Design principles for highly efficient quadrupeds and implementation on the MIT Cheetah robot," Proceedings - IEEE International Conference on Robotics and Automation, pp. 3307-3312, 2013, doi: 10.1109/ICRA.2013.6631038.

[4] G. Carbone, L. Mangialardi, and G. Mantriota, "A comparison of the performances of full and half toroidal traction drives," Mechanism and Machine Theory, vol. 39, no. 9, pp. 921-942, 2004, doi: 10.1016/j.mechmachtheory.2004.04.003.

[5] M. Nakano, H. Kumura, J. Sugihara, H. Mori, and N. Maruyama, "Development of a large torque capacity half-toroidal CVT," SAE Technical Papers, vol. 2000, no. 724, 2000, doi: 10.4271/2000-01-0825.

[6] J. Kim, F. C. Park, Y. Park, and M. Shizuo, "Design and analysis of a spherical continuously variable transmission," Journal of Mechanical Design, Transactions of the ASME, vol. 124, no. 1, pp. 21-29, 2002, doi: 10.1115/1.1436487. 
[7] C. Everarts, B. Dehez, and R. Ronsse, "Novel infinitely Variable Transmission allowing efficient transmission ratio variations at rest," IEEE International Conference on Intelligent Robots and Systems, vol. 2015-Decem, pp. 5844-5849, 2015, doi: 10.1109/IROS.2015.7354207.

[8] A. S. Kembaum, M. Kitchell, and M. Crittenden, "An ultra-compact infinitely variable transmission for robotics," Proceedings - IEEE International Conference on Robotics and Automation, pp. 18001807, 2017, doi: 10.1109/ICRA.2017.7989212.

[9] N. G. Park, J. H. Ryu, H. W. Lee, Y. H. Jeon, and N. Zhang, "Development of the Inner Spherical CVT for a motorcycle," International Journal of Automotive Technology, vol. 10, no. 3, pp. 341-346, Jun. 2009, doi: 10.1007/s12239-009-0039-8.

[10] H. Ghariblu, A. Behroozirad, and A. Madandar, "Traction and Efficiency Performance of Ball Type CVTs," International Journal of Automotive Engineering, vol. 4, no. 2, pp. 738-748, 2014.

[11] N. Srivastava and I. Haque, "A review on belt and chain continuously variable transmissions (CVT): Dynamics and control," Mechanism and Machine Theory, vol. 44, no. 1, pp. 19-41, 2009, doi: 10.1016/j.mechmachtheory.2008.06.007.

[12] M. Tomaselli, F. Bottiglione, P. Lino, and G. Carbone, "NuVinci drive: Modeling and performance analysis," Mechanism and Machine Theory, vol. 150, p. 103877 , 2020, https://doi.org/10.1016/j.mechmachtheory.2020.10387 7.

[13] T. Verstraten et al., "Modeling and design of an energy-efficient dual-motor actuation unit with a planetary differential and holding brakes," Mechatronics, vol. 49, no. December 2017, pp. 134148, 2018, doi: 10.1016/j.mechatronics.2017.12.005.

[14] D. Rabindran and D. Tesar, "Parametric design and power-flow analysis of parallel force/velocity actuators," Journal of Mechanisms and Robotics, vol. 1, no. 1, pp. 1-10, 2009, doi: 10.1115/1.2959100.

[15] A. Girard and H. H. Asada, "A two-speed actuator for robotics with fast seamless gear shifting," IEEE International Conference on Intelligent Robots and Systems, vol. 2015-Decem, pp. 4704-4711, 2015, doi: 10.1109/IROS.2015.7354047.

[16] P. López-García, S. Crispel, T. Verstraten, and E. Saerens, "Wolfrom Gearboxes for Lightweight , Human-Centered Robotics," no. September, 2019.

[17] A. Kapelevich, "High gear ratio epicyclic drives analysis," American Gear Manufacturers Association Fall Technical Meeting 2013, no. June, pp. 61-72, 2013.

[18] S. Crispel et al., "Introducing Compound Planetary Gears (C-PGTs): A Compact Way to Achieve High Gear Ratios for Wearable Robots," in International Symposium on Wearable Robotics, vol. 22, no. 337596, Springer, 2019, pp. 485-489.

[19] T. Iino et al., "Research of hydrostatic CVT for passenger vehicles," JSAE Review, vol. 24, no. 2, pp. 227-230, 2003, doi: 10.1016/S0389-4304(03)00002$\mathrm{X}$.

[20] C. Zhu, H. Liu, J. Tian, Q. Xiao, and X. Du, "Experimental investigation on the efficiency of the pulley-drive CVT," International Journal of Automotive Technology, vol. 11, no. 2, pp. 257-261, Apr. 2010, doi: 10.1007/s12239-010-0032-2.

[21] Nissan USA, "Nissan's XTRONIC Continuously Variable Transmission (CVT)," 2017. https://www.nissanusa.com/blog/xtronic-cvtcontinuously-variable-transmission.

[22] D. A. Winter, "Biomechanical motor patterns in normal walking," Journal of Motor Behavior, vol. 15, no. 4, pp. 302-330, 1983, doi: $10.1080 / 00222895.1983 .10735302$.

[23] T. Verstraten, R. Furnémont, P. López-García, D. Rodriguez-Cianca, B. Vanderborght, and D. Lefeber, "Kinematically redundant actuators, a solution for conflicting torque-speed requirements," The International Journal of Robotics Research, vol. 38, no. 5, pp. 612-629, 2019, doi: $10.1177 / 0278364919826382$.

[24] D. Yu and N. Beachley, "On the Mechanical Efficiency of Differential Gearing," Journal of Mechanisms, Transmissions, and Automation in Design, vol. 107, no. 1, pp. 61-67, 1985, doi: 10.1115/1.3258696.

[25] M. de Carlo and G. Mantriota, "Electric vehicles with two motors combined via planetary gear train," Mechanism and Machine Theory, vol. 148, p. 103789, 2020, doi: 10.1016/j.mechmachtheory.2020.103789.

[26] T. Verstraten, R. Furnémont, P. López-García, S. Crispel, B. Vanderborght, and D. Lefeber, "A series elastic dual-motor actuator concept for wearable robotics," Biosystems and Biorobotics, vol. 22, pp. 165-169, 2019, doi: 10.1007/978-3-030-01887-0 32.

[27] J. B. Morrell and J. K. Salisbury, "Parallel-coupled micro-macro actuators," International Journal of Robotics Research, vol. 17, no. 7, pp. 773-791, Jul. 1998, doi: 10.1177/027836499801700707.

[28] G. Mathijssen et al., "+SPEA introduction: Drastic actuator energy requirement reduction by symbiosis of parallel motors, springs and locking mechanisms," Proceedings - IEEE International Conference on Robotics and Automation, vol. 2016-June, pp. 676681, 2016, doi: 10.1109/ICRA.2016.7487193.

[29] J. M. Miller, "Hybrid electric vehicle propulsion system architectures of the e-CVT type," IEEE Transactions on Power Electronics, vol. 21, no. 3, pp. 756-767, 2006, doi: 10.1109/tpel.2006.872372.

[30] J. Schultz, G. Mathijssen, B. Vanderborght, and A. Bicchi, "A selective recruitment strategy for exploiting muscle-like actuator impedance properties," IEEE International Conference on Intelligent Robots and Systems, vol. 2015-Decem, pp. 2231-2237, 2015, doi: 10.1109/IROS.2015.7353676.

[31] H. W. Müller, Epicyclic drive trains: Analysis, synthesis, and applications. Wayne State University Press, 1982.

[32] G. Mathijssen et al., "Study on electric energy consumed in intermittent series-parallel elastic actuators (iSPEA)," Bioinspiration \& Biomimetics, vol. 12, no. 3, p. 036008, Apr. 2017, doi: 10.1088/1748-3190/aa664d.

[33] Quora, "Which Transmission is better, an automatic or a CVT?" https://www.quora.com/Which-transmissionis-better-an-automatic-or-a-CVT. 